\title{
Europäisierung vor Ort. Eine Ethnographie der Frontex- Mission Nautilus II auf dem Mittelmeer
}

Der Artikel präsentiert die Ergebnisse einer ethnographischen Feldforschung in Malta zur Frontex-Mission Nautilus II auf dem Mittelmeer. Er skizziert zunächst die Entstehung der europäischen Grenzschutzagentur Frontex und ordnet sie in die allgemeinen Entwicklungen der EU-Politik an den Außengrenzen ein. Anschließend wird die Ethnographie der Seemission Nautilus II präsentiert, die hauptsächlich aus Interviewmaterial mit an der Mission beteiligten Kommandeuren entstanden ist. Eine Hauptfrage ist dabei, wie das erklärte Ziel, die MigrantInnen von der Anlandung auf europäischem Territorium abzuhalten, mit dem europäischen Flüchtlingsschutz und dem Gebot des Non-Refoulement zusammengeht. Mithilfe einer rechtsanthropologischen theoretischen Perspektive zeigt der Artikel, welche Auswirkungen die Europäisierung des Grenzschutzes hat und welche Rechtsunsicherheiten entstanden sind. Der rechtliche Spielraum der Sicherheitskräfte, die Intransparenz der Mission, aber auch Handlungslogiken, die sich im Grenzraum ergeben, führen zu informellen und illegalen Praktiken innerhalb der Frontex-Mission, die nicht mit Flüchtlingsrecht konform gehen. Abschließend werden Fragen der europäischen Integration und Harmonisierung des Flüchtlingsschutzes diskutiert.

Schlagworte: Frontex, Malta, legal anthropology, Flüchtlinge, Mittelmeer

\section{Frontex und die Aushandlung von Flüchtlingsschutz auf dem Mittelmeer}

Durch die Europäisierung des Flüchtlingsschutzes und der Grenzkontrollen seit den 1990er Jahren und die Verfolgung neuer Ansätze im Grenzschutz wie dem sogenannten Integrated Border Management haben sich Entwicklungen ergeben, die für Flüchtlinge im Grenzraum des Mittelmeeres besonders starke Auswirkungen haben: Der Zugang in die Länder der EU und damit der Zugang zu einem Asylverfahren ist immer schwieriger geworden. Zudem ist unklar, wo und inwieweit der Grundpfeiler des internationalen Flüchtlingsrechts, das Gebot des Non-Refoulement (Art. 33 der Genfer Flüchtlingskonvention von 1951), auf See Gültigkeit hat. Dieses Gebot untersagt es, Personen in einen Staat zurückzuweisen, wo sie schwerwiegenden Menschenrechtsverletzungen ausgesetzt wären und enthält die Verpflichtung, ihnen Zugang zur Prüfung ihres Asylbegehrens zu gewähren. Besonders bei 
den gemeinsamen Patrouillen auf dem Mittelmeer der europäischen Grenzagentur Frontex ist strittig, wer für aufgegriffene MigrantInnen und ihre Asylgesuche verantwortlich ist. Die Mitgliedsländer der Europäischen Union sind zudem bei der Handhabung und Durchführung von Kontrollen auf dem Mittelmeer, den sogenannten interceptions, tief gespalten. Besonders deutlich werden die rechtlichen und politischen Konflikte bei den Frontex-Missionen, die den Auftrag haben, die gemeinsamen Außengrenzen der EU zu kontrollieren. ${ }^{1}$

Der Artikel stellt Ausschnitte einer länderübergreifenden Forschung im Grenzraum des Mittelmeers in Libyen, Malta und Süditalien in den Jahren 2006 bis 2008 vor (Klepp 2011 a). Die Studie verbindet die empirische Forschung von Grenzregionen mit einer rechtsanthropologischen theoretischen Perspektive. Sie untersucht die Effekte von (Grenz-)Räumen auf die Entwicklung von Flüchtlings- und Menschenrechten. Vor Ort im Grenzgebiet zeigt sich, dass trotz der Harmonisierung des EU-Flüchtlingsrechts keineswegs nur die EU-Gesetzgebung bei der Umsetzung des Rechts bedeutsam ist. Auch lokale und nationale Interessen, die den europäischen Regelungen zum Teil zuwiderlaufen, werden hier relevant. Mein Interesse gilt daher den verschiedenen AkteurInnen im europäischen Flüchtlingsschutz und ihren Verknüpfungen auf der lokalen, nationalen und supranationalen Ebene. Denn in deren Zusammenspiel, so die These, konstituieren sich die europäische Flüchtlingspolitik und ihre formellen und informellen Aushandlungsprozesse.

Im ersten Teil des Artikels wird die institutionelle Entwicklung von Frontex nachgezeichnet und in die allgemeineren Entwicklungen der EU-Politik an den Außengrenzen eingeordnet. Anschließend wird im zweiten Teil beschrieben, welche rechtlichen und operativen Konstellationen und Probleme auf dem Mittelmeer auftreten, wenn BootsmigrantInnen von Frontex-Missionen innerhalb von Grenzschutzaktivitäten abgefangen werden sollen: Wie gehen die aus verschiedenen Sicherheitskräften der Mitgliedsländer zusammengesetzten Missionen bei dem Aufbringen der MigrantInnenboote auf See vor? Wie geht insgesamt das erklärte Ziel, die MigrantInnen von der Anlandung auf europäischem Territorium abzuhalten, mit dem Hauptpfeiler des europäischen Flüchtlingsschutzes, dem Gebot des Non-Refoulement, zusammen? Es wird anhand der Nautilus II-Mission gezeigt, welche Folgen die rechtlichen Unklarheiten auf der operationellen Ebene auf See haben und in welcher Weise die Sicherheitskräfte der Mitgliedsländer damit umgehen. Durch ihre zentrale Position im Aushandlungsprozess um Flüchtlingsschutz und Grenzkontrolle, ihren großen rechtlichen Spielraum aufgrund fehlender Regulierungen, aber auch aufgrund von Zwängen und Handlungslogiken, die sich im Grenzraum ergeben, wird europäische Grenz- und

1 Dieser Artikel ist Keebet und Franz von Benda-Beckmann gewidmet. 
Flüchtlingspolitik von Sicherheitskräften maßgeblich bestimmt. Recht wird von den Hoheitsvertretern der Staaten damit nicht nur umgesetzt, sondern auch geschaffen (vgl. Lipsky 1980). Zudem können Verantwortlichkeiten für Asylgesuche, die bei nationaler Regelung des Asylrechts klar waren, durch die Europäisierung des Grenzschutzes undeutlich werden. Abschließend wird die Rolle der Agentur Frontex beim Menschen- und Flüchtlingsschutz auf dem Mittelmeer analysiert.

\section{Frontex - eine europäische Agentur konstituiert sich}

Mit der Verordnung (EG) Nr. 2007/2004 gründet die Europäischen Union am 26. Oktober 2004 die Agentur mit dem lautmalerischen Namen »Frontex« (vom französischen frontière exterieure): die Europäische Agentur für die operative Zusammenarbeit an den Außengrenzen. Der Beschluss dieser sogenannten FrontexVerordnung (Frontex-VO) markiert auch den Beginn der Institutionalisierung der europäischen Grenzsicherung. Die Institutionalisierung des europäischen Grenzschutzes mit der Schaffung einer Agentur ist dabei ein Kompromiss: zwischen den Mitgliedstaaten, die auf ihre nationalstaatliche Souveränität, jedoch auch auf Synergien bei der Nutzung und Entwicklung technischen Geräts zur Grenzüberwachung und beim Schutz der EU-Außengrenzen bedacht sind, und der EU-Kommission, die auf ein gemeinsames Vorgehen und Solidarität im Grenzschutzbereich drängt (Leonard 2009: 371-388; Kasparek 2010: 113). Frontex erhält unter anderem durch den Auftrag, ein einheitliches Vorgehen an den EU-Außengrenzen aufgrund einer gemeinsamen rechtlichen Basis zu schaffen, eine machtvolle Position (European Council on Refugees and Exiles 2007: 11). Es sollen rechtliche Fragen konkretisiert und Kernentscheidungen für die Zukunft der EU-Grenzsicherung und damit für den EU-Flüchtlingsschutz getroffen werden. Der operative Betrieb der Grenzschutzagentur wurde am 3. Oktober 2005 aufgenommen, ihr Sitz liegt in Warschau. Laut Verordnung hat Frontex vor allem die Aufgabe,

- die operative Zusammenarbeit der Mitgliedstaaten im Bereich der Außengrenzen zu koordinieren (Art. 3 der Frontex-VO);

- Risikoanalysen zu erstellen (Art. 4 der Frontex-VO);

- die EU-Länder bei der Ausbildung von Grenzschutzbeamten zu unterstützen und gemeinsame Normen festzulegen (Art. 5 der Frontex-VO);

- die Mitgliedstaaten in Situationen zu unterstützen, die eine verstärkte technische und operative Unterstützung an den Außengrenzen erfordern (Art. 8 der Frontex$\mathrm{VO}$ );

- gemeinsame Rückführungsaktionen der Mitgliedsländer zu koordinieren und zu unterstützen (Art. 9 der Frontex-VO). 
Über diese Aufgaben hinaus soll Frontex die Zusammenarbeit mit nationalen Geheimdiensten suchen und kann in der Kooperation mit den Partnereinrichtungen $O L A F$ (Europäisches Amt zur Betrugsbekämpfung), Eurojust (Einheit für justizielle Zusammenarbeit der Europäischen Union) und Europol (Europäisches Polizeiamt) auf deren Datenbanken zugreifen. In Artikel 13 und 14 der Frontex-VO ist zudem festgelegt, dass Frontex eigenständige Kooperationen und Arbeitsabkommen mit Institutionen von Drittländern und Internationalen Organisationen eingehen kann. Die weitreichenden Kompetenzen in der Zusammenarbeit mit Dritten wurden dabei von den Mitgliedsländern teilweise als Eingriff in die hoheitlichen Aufgaben kritisch bewertet (Jeandesboz 2008: 12).

Seit 2005 wird Frontex mit den sogenannten »Frontex-Missionen« vor Ort aktiv. Verschiedene Risikoanalysen, die für die Einsätze von Frontex zentral sind, konnten ebenfalls im ersten Jahr des Bestehens vorgelegt werden (Frontex 2006). Im Jahr 2006 wurden neben einer Vielzahl von anderen Aktivitäten 15 gemeinsame Missionen an See- und Landesgrenzen und auch an Flughäfen durchgeführt (Frontex 2007 a). Dabei sind Ausstattung, Budget und zeitlicher Umfang der Missionen sehr unterschiedlich. Frontex hat keine eigenen Soldaten, sondern setzt sich aus den teilnehmenden Nationen an einzelnen Missionen und deren Streitkräften zusammen. Grundsätzlich gilt, dass Frontex auf die in der sogenannten toolbox von den Mitgliedsländern bereitgestellten Gerätschaften zurückgreifen kann. Die Liste mit dem offiziellen Namen Centralised Records of Available Technical Equipment for Control and Surveillance of External Borders (CRATE) umfasste im September 2007 115 Schiffe, 27 Hubschrauber, 21 Flugzeuge und weiteres Material zur Überwachung von Grenzen (Kasparek/Marischka 2008: 45). Weil es immer wieder Konflikte um die tatsächliche Bereitstellung der von den Staaten angegebenen Gerätschaften gibt, kann Frontex seit 2011 auch eigenes Material anschaffen. Da die Möglichkeiten der Frontex-VO, Grenzbeamte auf Anforderung in Notfallsituationen innerhalb kürzester Zeit einzusetzen, zu gering erschienen, wurde 2007 die Ausweitung der Frontex-VO beschlossen und die Rapid Border Intervention Teams (RABITS) ins Leben gerufen (Verordnung (EG) Nr. 863/2007). Dies sind Verbände von Grenzsoldaten und Spezialisten, die in Notfallsituationen an den EUAußengrenzen eingesetzt werden können. Innerhalb von nur zehn Tagen nach Anforderung sollen sie vor Ort sein.

Ein weiterer wichtiger Aspekt der Arbeit der Agentur ist die zunehmende digitale, auch biometrische Kontrolle an den europäischen Grenzen, die mit dem Einsatz neuer Technologien einhergeht, und die Unterstützungs- und Vernetzungsaufgabe, die Frontex hier übernimmt. Mit der Schaffung von EUROSUR (European External Border Surveillance System), eines Europäischen Grenzkontrollsystems (KOM 
2008: 68), wird die Vernetzung aller bestehenden Grenzüberwachungstechnologien angestrebt. Frontex soll bei diesen Entwicklungen federführend sein und mit neuen Technologien die »feinkörnige, individualisierte Kontrolle der Bevölkerung Schengenlands erlauben « (Kasparek 2010: 124). Das Budget der Agentur ist rasch gewachsen. 2006 wurde es von 12,4 auf 19,2 Millionen Euro erhöht (European Council on Refugees and Exiles 2007: 12). 2011 lag das Budget bei ca. 88 Millionen Euro. Aktuell liegen die Hauptaktivitäten der Frontex-See-Missionen bei den Missionen Poseidon und einer RABIT-Operation (November 2010 bis Februar 2011) im östlichen Mittelmeer, vor allem in Griechenland an der Grenze zur Türkei. Zudem gab es 2011 die Hermes-Mission auf der italienischen Insel Lampedusa im zentralen Mittelmeer (Frontex 2013).

Im Vergleich zu verschiedenen Institutionen der EU, die bis in die 1990er Jahre geschaffen wurden und eher den Charakter von Foren zum Austausch und zur Informationsvernetzung hatten, verfügt Frontex über einen weitreichenden operativen Auftrag und hoheitliche Befugnisse. Wie Andreas Fischer-Lescano und Timo Tohidipur bemängeln, steht diesen Befugnissen jedoch keine ausreichende parlamentarische Kontrolle oder eine politische Einflussnahme auf die Agentur von Rat und Kommission gegenüber. Das EU-Parlament hat lediglich die Aufgabe, das Budget von Frontex mitzubestimmen, jedoch keine direkte Kontrollmöglichkeit. Die Gründung von Agenturen mit solch weitreichenden Möglichkeiten könnte in der Konsequenz eine Verselbstständigung des administrativen Apparates bedeuten (Fischer-Lescano/Tohidipur 2007: 21). Die Grenzschutzagentur wird von einem Exekutivdirektor geleitet, der vom Verwaltungsrat der Agentur auf Vorschlag der EU-Kommission ernannt wird. Der Verwaltungsrat setzt sich aus Vertretern der Mitgliedsländer sowie der anderen Schengen-Länder und zwei Vertretern der EUKommission zusammen. Er fällt seine Entscheidungen mit der absoluten Mehrheit seiner Mitglieder.

Nach Auffassung von Fischer-Lescano und Tohidipur (2007: 20) ist die Leitung von Frontex dabei ein Beispiel für die Integration der Exekutive in die Agentur und für die mangelnde Kontrolle der Agentur außerhalb ihrer eigenen Strukturen. Julien Jeandesboz (2008: 15) kritisiert zudem, dass auch die Evaluierung der FrontexAktivitäten durch die EU-Kommission große Schwächen habe und zu sehr auf technische Fragen und allgemeine Effizienz fokussiere; Auswirkungen dieser Aktivitäten auf die Freiheit und grundlegenden Rechte von Menschen würden hingegen nicht thematisiert. Insgesamt wird bemängelt, dass das EU-Parlament mit seiner Rolle als starker Vertreter der Menschenrechte bei der Schaffung von Frontex zu wenig eingebunden worden sei. Nur der Entwurf der Frontex-VO wurde im Parla- 
ment diskutiert, nicht jedoch über die endgültige Verordnung abgestimmt (Leonard 2009: 381).

In der kurzen Zeit ihres Bestehens konnte die Agentur auf verschiedenen Ebenen ein weitgefächertes Spektrum an Aktivitäten entfalten, was auch am Wachstum des Frontex-Budgets liegt, das sich in wenigen Jahren vervielfacht hat. Derzeit vereint Frontex »Elemente von Grenzschutzpolizei, Geheimdienst, Ausbildungsinstitut und Forschungseinrichtung «, wie es Bernd Kasparek (2010: 114) beschreibt, und kann als Experimentierfeld und »think tank« zukünftiger europäischer Grenzsicherung dargestellt werden. Ob die Entwicklungen eine »Europäische Grenzpolizei« möglich machen, ist jedoch weiter offen. Im Vorfeld der Gründung wurde diese Option von den Mitgliedsländern ausdrücklich abgelehnt (Jeandesboz 2008: 12).

Die Arbeit von Frontex schließt insgesamt an die Entwicklungen in der EUGrenz- und Migrationspolitik an, welche die Schaffung eines Integrated Border Management (IBM) vorsehen. Jeder Gefahr, die über die Außengrenzen in die EU gelangen könnte, soll hierbei mit einem entsprechend vielschichtigen Maßnahmenkatalog begegnet werden. Solidarität unter den Mitgliedsländern, gemeinsame rechtliche Regelungen und gemeinsame Operationen der Sicherheitskräfte gehören zu den Komponenten des IBM und fallen in den Aufgabenbereich der Agentur Frontex. Auf einem europäischen Polizeikongress im Februar 2007 wurde das Stufenmodell, das auf die Durchführung von Kontrollen und auf die Einflussnahme der EU in einem ausgedehnten Grenzraum abzielt, von Frontex vorgestellt (European Council on Refugees and Exiles 2007: 20): ${ }^{2}$ Die erste Stufe der Kontrolle sieht dabei schon im Herkunftsland die Zusammenarbeit mit Verbindungsbeamten und Kooperationsabkommen vor, die möglichst früh irreguläre Migrationsbewegungen in Richtung Europa verhindern sollen. Die zweite Stufe, Across the Border genannt, zielt auf die Kooperation mit den direkten Nachbarländern der Europäischen Union ab. Die dritte Stufe betrifft schließlich die Kontrollen an den eigenen Außengrenzen und die vierte Stufe der Grenzüberwachung findet im EU-Binnenraum statt. Auch das Haager Programm von 2004, das bis 2010 die Politik der EU im Bereich Grenzund Migrationspolitik bestimmte, und die anschließenden Ratstreffen der EU machten die Zusammenarbeit mit Herkunfts- und Transitländern und die verstärkte Ko-

2 Ein ähnliches vierstufiges Modell zum Schutze der EU-Außengrenzen findet sich schon im $E U$ Schengen-Katalog 2002: Kontrollen an den Außengrenzen, Abschiebung und Rückübernahme: Empfehlungen und bewährte Praktiken, vom 28.2.2002. Auch die Mitteilung der Kommission an den Rat und das europäische Parlament (KOM (2002) 233 endgültig) mit dem Titel: »Auf dem Weg zu einem integrierten Grenzschutz an den Außengrenzen der EU-Mitgliedstaaten« beschäftigt sich mit diesem Modell. 
operation unter den Mitgliedstaaten zu den zentralen Pfeilern der EU-Politik an den Außengrenzen.

Mit diesen Entwicklungen haben sich die ehemals als Linien gedachten und konzipierten Grenzen zu einem Grenzraum gewandelt, der weit in das nationalstaatliche Territorium der Mitgliedsländer und in das der Drittländer außerhalb der EU hineinreicht. Diese Neudefinition von Grenzen wird seit einigen Jahren unter dem Begriff der »Exterritorialisierung« der EU-Grenz- und Migrationspolitik diskutiert (vgl. zum Beispiel Lavenex/Ucarer 2004: 417-443). Durch die enge diskursive Verknüpfung von Migrationsfragen mit den Bereichen Sicherheit, Terrorismus und EUAußengrenzen, vor allem nach den Anschlägen des 11. Septembers 2001, wurde Migration, und nicht nur undokumentierte Migration, zudem zunehmend als Sicherheitsrisiko konzeptualisiert und damit kriminalisiert. Dies gilt vor allem für das Phänomen der Mittelmeermigration (Carrera 2007: 6). Insgesamt kann Frontex sowohl als federführende Kraft als auch als Produkt der Entwicklungen bezeichnet werden.

Nach einem Überblick zu der Entwicklung von Frontex und einer Skizzierung von Veränderungen, die sich durch die Europäisierung der Grenzsicherung ergeben, sollen nun die operativen Missionen von Frontex auf dem Mittelmeer zur Sprache kommen, die besonders in das Interesse der Öffentlichkeit rückten und in die sich auch die von mir untersuchte Nautilus II-Mission aus dem Jahre 2007 einfügt.

\section{Nautilus $I I-»$ We have no idea where they are going ...»}

In allen Papieren der EU-Institutionen, die in den letzten Jahren zum Umgang mit der südlichen Seegrenze produziert wurden, wird die Bedeutung betont, die der Ausbau der südlichen Seegrenze für die Entwicklung eines gemeinsamen integrierten Grenzschutzsystems habe (siehe zum Beispiel KOM 2006: 12). Zentral für die Entwicklung des EU-Grenzschutzsystems sei dabei die Solidarität unter den Mitgliedstaaten, vor allem mit den am stärksten von irregulärer Migration betroffenen EU-Mittelmeeranrainern. Als wichtigstes Instrument zum gemeinsamen Grenzschutz wird regelmäßig der Ausbau der Frontex-Kapazitäten genannt. Die schnelle Entwicklung der Agentur und die Steigerung des Frontex-Budgets in wenigen Jahren um ein Vielfaches trägt den großen Erwartungen an Frontex Rechnung. Die offenen rechtlichen Probleme, die bei der gemeinsamen Überwachung der EU-Seegrenzen durch Frontex aufgetaucht sind, kreisen vor allem um die Frage, wie beim Abfangen und Aufbringen von MigrantInnenbooten zu verfahren sei, da »an diesen Operationen Kräfte verschiedener Mitgliedstaaten teilnehmen, die sich nicht immer über Art und Zeitpunkt des Abfangens einig sind« (KOM 2006: 12). 
Im Folgenden möchte ich zunächst einige methodische Aspekte meiner Studie offen legen. Anschließend werde ich auf das politische Klima in Malta bezüglich der Bootsflüchtlinge eingehen. Die bedrängte »Frontstaatenperspektive« des Frontex-Gastlandes Malta, das sich als »Bollwerk gegen die afrikanische Migration « ${ }^{3}$ sieht, spielt innerhalb der Frontex-Mission Nautilus II eine entscheidende Rolle. Daran anschließend wird die Ethnographie der Frontex-Mission Nautilus II präsentiert. Dabei wird illustriert, zu welchen rechtlichen und operativen Schwierigkeiten die noch jungen Frontex-Missionen im Mittelmeer führen. Die rechtlichen Unstimmigkeiten auf europäischer Ebene setzten sich dabei in den Frontex-Missionen auf See fort. Ohne praktische Leitlinien und einheitliche Regelungen für den Umgang mit MigrantInnen auf See haben die Missionen im Jahr 2006 dennoch ihre praktische Arbeit in gemeinsamen Einsätzen auf dem Mittelmeer aufgenommen. Die rechtlichen Lücken sind mit gravierenden Unsicherheiten für die Sicherheitskräfte vor Ort verbunden. Diese Unklarheiten bieten jedoch auch einen Handlungsspielraum, der von den Beamten im nationalen Interesse der einzelnen Länder ausgefüllt wird, wie meine Forschung zeigt.

\subsection{Methodische Aspekte}

Um den Grenzraum des Mittelmeers, die Begebenheiten auf See und deren Implikationen für den Flüchtlingsschutz zu untersuchen, ist der einzig gangbare Weg eine Annäherung von den Küsten aus. Meine länderübergreifende Feldforschung umfasste einen fünfwöchigen Aufenthalt in Libyen im Oktober und November 2006; in Süditalien habe ich von März bis August 2007 geforscht und in Malta im September und Oktober 2007 (vgl. Klepp 2011 a). In der Ethnographie soll möglichst unterschiedlichen Perspektiven im Feld Rechnung getragen werden, um die Beziehungsgeflechte vor Ort, den Aushandlungsprozess zwischen »Lokalem« und »Globalem « adäquat zu untersuchen. Meine Forschung in Malta beschreibt, in welche Situation die fehlende oder sehr weit gefasste rechtliche Basis der Frontex-Missionen die Sicherheitskräfte bringt und welche Perspektiven diese wiederum in die Missionen einbringen. Dadurch werden die Wechselwirkungen in den europäischen Rechtsentwicklungen, die aus unterregulierten Situationen im Grenzraum entstehen, deutlich. Es ist jedoch schwierig, den Deckmantel der Unklarheit, der über den

3 Interview mit Mario Abela, Sprecher des Innenministeriums, am 18.9.2007 in einem Saal des Ministeriums. Mit der Änderung aller Namen in diesem Artikel soll ein gewisses Maß an Anonymisierung erreicht werden. Wichtige Amtsbezeichnungen wurden jedoch beibehalten. Alle Interviewten haben einer Veröffentlichung ihrer Äußerungen in wissenschaftlichen Publikationen zugestimmt. Ich zitiere nachfolgend aus meinen Interviewtranskripten; die von mir gestellten Fragen sind kursiv gesetzt. 
Geschehnissen auf dem Meer liegt, zu lüften. Die Forschung auf Malta war durch den begrenzten Zugang zu den Grenzanlagen und der Unmöglichkeit der direkten Beobachtung der Praktiken auf See stark eingeschränkt. Weder konnte eine teilnehmende Beobachtung auf See während der Grenzpatrouillen realisiert werden, noch gab es Zugang zu MigrantInnen, die von Frontex-Missionen aufgegriffen worden waren. Sie wurden in den Haftzentren auf Malta festgehalten, zu welchen ich keinen Zugang erhalten konnte.

Die sogenannten Nautilus-Operationen patrouillierten seit 2006 im zentralen Mittelmeer zwischen Italien, Malta und Libyen. Für die Auswahl der Mission Nautilus II für meine Studie war entscheidend, dass ich Zugang zu der Mission erhalten konnte. Ein für mich glücklicher Zufall war dabei, dass die Frontex-Mission im gleichen Zeitraum durchgeführt wurde wie mein Feldaufenthalt. Da die Einsätze geheim gehalten werden, war dies nicht planbar. Zentral waren bei der Datenerhebung in Malta offene, zum größten Teil leitfadengestützte Interviews mit Kommandeuren der Mission, die ich bei der maltesischen Armee angefragt hatte. Die Forschung wurde von mir allein durchgeführt. Für den vorliegenden Artikel war neben meiner Forschung in Malta vor allem die Analyse der EU-Dokumente im Bereich der Mittelmeermigration sowie der rechtlichen Basis der Europäisierung des Grenz- und des Flüchtlingsschutzes relevant.

Insgesamt habe ich in Malta 16 Interviews mit Angestellten der Armed Forces of Malta (AFM) und des Innenministeriums, mit Mitarbeitern von Flüchtlingsorganisationen wie UNHCR und Jesuit Refugee Service sowie mit weiteren AkteurInnen durchführen können. Drei in die Frontex-Mission involvierte AFM-Kommandeure waren zu Interviews mit mir bereit, zudem ein maltesischer Geheimdienstmitarbeiter, der für die Mission abgestellt war. Die Interviews wurden aufgezeichnet. Sie gaben Einblick in einen Bereich, der durch ein Informations- und Kontrollmonopol der Sicherheitskräfte geprägt und damit für Dritte zumeist unzugänglich ist. Die »Wahrheit« über die Geschehnisse auf See ist damit nicht zu erfahren. Die Perspektiven der maltesischen Kommandeure und Beamten, ihre zum Teil widersprüchlichen Angaben und Erzählungen, geben jedoch Auskunft über einige Aspekte der Handlungen von Sicherheitskräften im Grenzraum und ihre Auswirkungen auf lokaler, nationaler und europäischer Ebene. Sie verdichten sich zu einem fragmentierten, jedoch aufschlussreichen Bild der Lage auf See und der FrontexEinsätze. Dabei soll ein moralisierendes, schwarz-weiß gezeichnetes Bild des Forschungsfeldes und eine vermeintlich klare Definition der Machtverhältnisse durch ein Täter-Opfer-Schema vermieden werden. Der Einblick in die Praktiken des Grenzraums soll vielmehr ermöglichen, die Handlungslogiken der unterschiedlichen Akteure zu verstehen und aufeinander zu beziehen. Die ethnographische Dar- 
stellung der Zustände im Grenzraum soll somit auch Chancen für eine verantwortliche Politik erschließen (vgl. Bourdieu 1997: 214).

\subsection{Malta und die »Frontstaatenperspektive»}

Malta ist mit 400.000 Einwohnern auf $316 \mathrm{~km}^{2}$ der kleinste Staat der Europäischen Union und zudem das am zweitdichtesten besiedelte Land der Welt. Als südlichstes Mitgliedsland der EU liegt es auf den Migrationsrouten, die über Libyen nach Italien und in die EU führen. Zwischen 2002 und 2008 sind auf Malta ungefähr 10.700 MigrantInnen angelandet. Scheinen diese Zahlen für andere Länder der EU gering zu sein, so bestimmen sie doch auf Malta einen großen Teil des medialen und politischen Geschehens. Hinsichtlich der Ängste, welche die Anlandungen bei der maltesischen Bevölkerung auslösen, schreibt Mariella Micaleff: »Invaded and dominated by outsiders for centuries, this new phenomenon has left the locals feeling threatened and increasingly hostile towards the irregular immigrants present on the island « (Micaleff 2006: 5). Und Fiona Texeire stellt sogar fest: »Nowadays, irregular migration in Malta has become a burning political issue, and has created a climate of psychosis« (Texeire 2006: 10). Ein Indiz für die Spannungen ist zum Beispiel die Tatsache, dass 2005 und 2006 neun Fahrzeuge des Jesuit Refugee Service Opfer von Brandanschlägen wurden. Wie auch bei weiteren Anschlägen auf FlüchtlingsanwältInnen und JournalistInnen wurden die Täter nicht gefasst. Der Sprecher des Innenministeriums sieht die Gründe für ein nahezu "psychotisches Klima« auf der Insel bezüglich der Anlandungen vor allem in der geringen Größe der Insel: »Because of our size people are seeing more of these immigrants. I don't call it xenophobia, but people are worried that we could be taken over. $\ll^{4}$

Auf meine Frage nach der Rolle Maltas für die EU-Grenzpolitik im Mittelmeer antwortet Jason St. John, ehemaliger Stratege des britischen Militärs und offizieller $»$ Berater der Regierung gegen irreguläre Migration «: $» W e$ are obviously in the frontline. We are obviously a front-line state and in strategic terms an important location. $\aleph^{5}$ Auf diese »Frontstaaten-Perspektive« der maltesischen Regierung im Umgang mit dem Phänomen der Seemigration, die eine Bedrohung der Insel durch BootsmigrantInnen impliziert, werde ich im Laufe des Artikels wieder zu sprechen kommen. Wie sich zeigt, kann diese Perspektive im Prozess der Europäisierung eine

4 Interview mit Mario Abela am 18.9.2007.

5 Interview mit Jason St. John am 2.10.2007 in La Valletta in einem Saal des Vereins Heritage Mal$t a$, für den St. John offensichtlich ehrenamtlich tätig ist. St. John hat einen einflussreichen Bericht zum Phänomen der Seemigration für die Regierung Maltas erstellt, dessen Empfehlungen nun Schritt für Schritt umgesetzt werden. 
wirkungsmächtige Rolle bei der Entwicklung des Flüchtlingsschutzes im Grenzraum und anderswo einnehmen, die über die nationalstaatlichen Grenzen Maltas weit hinausgehen.

\subsection{Offene rechtliche Fragen der Frontex-Mission -»There is no body of international law which covers it ....}

Schon im Jahr 2006 hatte es eine erste Nautilus-Mission vor Malta und Italien gegeben. Nautilus I (griechisch: der Segler) bestand zum einen aus dem Einsatz einer Expertengruppe aus verschiedenen Mitgliedsländern, welche die Identifikation der ankommenden MigrantInnen auf Malta und eine Erhöhung der Rückführungsrate in die Heimatländer erreichen sollte. Zum anderen fand zwischen dem 5. und 15. Oktober 2006 eine Seeaktion statt, an der Malta, Italien, Griechenland, Frankreich und Deutschland teilnahmen. Diese Aktion hatte zum Ziel »die illegalen Einwanderungsströme aus Malta und Lampedusa zu stoppen « (Frontex 2007 a: 14).

Die erste Phase der Nautilus II-Mission begann am 25. Juni und wurde am 27. Juli 2007 überraschend eingestellt. Der Referatsleiter für Politikentwicklung im maltesischen Innenministerium (Department for Policy Development) Andrew Mulvaney erzählt später, wie »geschockt« die Malteser gewesen seien, dass die Mission so plötzlich beendet wurde: »Mitten in der Hauptsaison der illegalen Migration! $\varkappa^{6}$ Die Mission musste wohl vor allem aus Geldmangel pausieren, da Mitgliedsländer bereits zugesagte Gelder für Frontex, auch zum Ärger des ehemaligen EU-Kommissars Franco Frattini, nicht freigemacht hatten. ${ }^{7}$ Während dieser ersten Phase von Nautilus II wurden innerhalb der sogenannten »operational area « 401 MigrantInnen auf See entdeckt (detected), weitere 63 außerhalb dieser Zone (Frontex 2007 b).

In Malta treffe ich im September 2007 Kommandeur Carmel Borg. Er ist für die operative Leitung der Marineeinsätze von AFM verantwortlich. Als Vertreter seines Landes reist er regelmäßig nach Brüssel, um an der Erstellung der Frontex-Leitlinien für Einsätze auf See mitzuwirken. Zunächst geht der Kommandeur, der versiert in Seerechtsfragen ist, da er einen einjährigen Kurs am Internationalen Seerechtsinstitut (IMLI) auf Malta besucht hat, auf verschiedene rechtliche Fragen der FrontexMission ein. Für den Seerechts-Experten ist klar, dass alle Frontex-Einsätze mit internationalem Seerecht konform gehen müssen. Doch auf dieser Ebene gebe es

6 Interview mit Andrew Mulvaney am 20.9.2007 in einem Saal des maltesischen Innenministeriums. Er legte großen Wert darauf, dass keine Tonbandaufzeichnungen von unserem Gespräch gemacht werden. Zitat aus Feldforschungsnotizen.

7 Interview mit Andrew Mulvaney am 20.9.2007. 
bei dem Aufbringen von MigrantInnenbooten innerhalb von Frontex-Einsätzen auf Hoher See Lücken, so der Kommandeur:

»The fact is, if you look at the current status of international law it doesn't really facilitate this type of interception operations. Because even if you look at some types of other laws, if you look at the Palermo protocols, they don't really apply here, about the nature, about the fact that they still believe in flag state permission and these are not wearing a flag, if you look at UNCLOS itself, the crimes which attract international jurisdiction are still slavery, piracy or illegal transmission. This is neither slavery or piracy or illegal transmission. Drug trafficking under the Vienna Convention could have been a possibility but that isn't drug trafficking either. There is no body of international law which covers it. $\ll^{8}$

Keine der internationalen Konventionen, die Borg anspricht ${ }^{9}$ und die direkt oder indirekt das Abfangen von Booten auf See betreffen, regeln, wie genau mit MigrantInnenbooten auf Hoher See zu verfahren ist. Zwar ist es laut UNCLOS (United Nations Convention on the Law of the Sea) möglich, ein unbeflaggtes Boot durch Sicherheitskräfte auch auf Hoher See aufzubringen, weil dann nicht das »Recht auf friedliche Durchfahrt $\aleph^{10}$ gilt. Doch wie Borg formuliert, habe Malta dazu eigentlich keine nationale Handhabe, könne also nur im Rettungsfall regulär eingreifen. Außerdem sei es äußerst gefährlich für alle Beteiligten, ein MigrantInnenboot gegen den Willen der Reisenden auf See anzuhalten. ${ }^{11}$ Er spielt auf einen Zwischenfall im Kanal von Otranto vor der italienischen Küste aus dem Jahr 1997 an. Hier waren bei dem Versuch der italienischen Marine, ein Flüchtlingsboot zu stoppen, 108 Menschen ertrunken. Der Kommandeur des Kriegsschiffes Sibilla wurde im März 2005 zu drei Jahren Haft verurteilt (OBC 2008).

8 Interview mit Carmel Borg, Kommandeur der AFM, am 22.9.2007 im Hauptquartier der Armee (Luqua Barracks).

9 Kommandeur Borg spricht hier verschiedene internationale Abkommen an, die sich direkt oder indirekt mit dem Abfangen von Schiffen oder Booten auf See befassen. Es geht um die Wiener Konvention gegen den illegalen Handel mit Suchtstoffen und psychotropen Substanzen von 1988, um das sogenannte »Palermo-Protokoll« (Zusatzprotokoll zur Verhütung, Bekämpfung und Bestrafung des Menschenhandels, insbesondere des Frauen- und Kinderhandels, zum Übereinkommen der Vereinten Nationen gegen die grenzüberschreitende organisierte Kriminalität) aus dem Jahr 2000 und um das Seerechtsabkommen UNCLOS von 1982.

10 Das »Recht auf friedliche Durchfahrt« ist ein wichtiges Konzept des internationalen Seerechts. Es beinhaltet das Recht, Küstenmeer und Archipelgewässer eines anderen Staates friedlich zu durchfahren und findet sich im Seerechtsübereinkommen der Vereinten Nationen von 1982.

11 Zur Problematik, MigrantInnenboote auf See auszubremsen, vergleiche auch Klepp (2007: 43-49). 
Am Ende unseres Gesprächs betont Borg nochmals, dass die allgemeine Mission von Frontex auf dem Mittelmeer aus seerechtlicher Sicht auf tönernen Füßen stehe: »The state of mission as an agency is to protect Europe's external borders, whether if they have an adequate legal background is the question, especially at sea. ${ }^{12} \mathrm{Die}$ komplexe rechtspluralistische Konstellation der europäischen Frontex-Einsätze, zusammengesetzt aus nationalstaatlichen Verbänden, die auch auf Hoher See operieren, weist somit nicht nur auf europäischer Ebene Lücken auf, die durch die fehlenden Einsatz-Leitlinien noch verstärkt werden. Das Aufbringen von MigrantInnenbooten auf See scheint auch durch internationales Recht nicht gedeckt. Wie aber gehen die Sicherheitskräfte mit dieser Rechtsunsicherheit um?

\subsection{Nautilus II trifft Entscheidungen - Die Schaffung von Recht durch street- level bureaucrats}

Von Andrew Fenek, koordinierender Kommandeur der Nautilus II-Mission, erhoffe ich mir einige Information zum Einsatz vor Ort. Fenek ist ebenfalls Marine-Kommandeur der AFM. Da Malta die Mission angefordert hat und Gastland von Nautilus II ist, ist er als maltesischer Kommandeur für diese Aufgabe abgestellt worden. Insgesamt sind vier Hochsee-Schiffe, sechs Küstenschiffe, drei Hubschrauber und vier Aufklärungsflugzeuge für Nautilus II, Phase II im Einsatz. Allerdings sind nicht immer alle Gerätschaften vor Ort. Am Tag unseres Interviews erwartet Kommandeur Fenek zwei Super Puma-Hubschrauber der deutschen Bundespolizei und ein griechisches Patrouillenboot. Neben Malta, Italien, Deutschland und Griechenland sind die EU-Mitgliedsländer Frankreich, Rumänien, Portugal und Großbritannien an der Mission beteiligt. Die Frontex-Agentur selbst habe ferner einen Nachrichtenoffizier und eine litauische Frontex-Koordinatorin aus Warschau geschickt.

Fenek erklärt, dass Frontex-Missionen zu Beginn des Jahres von Frontex geplant werden: »With specific dates in mind and the asset and then the member states decide which asset they allocate to this operation and than Frontex will do an assessment how much the operation will cost using this assets. And according to the budget it will modify the operation. $\ll^{13}$ Bis zu $80 \%$ der Kosten des Einsatzes würde Frontex direkt übernehmen, bis zu 20\% hingegen die Mitgliedsländer, die für Teilkosten ihrer eingesetzten Gerätschaften und Sicherheitskräfte aufkommen, so Fenek. Er betont, dass Frontex zwar zahle, aber die Zusammenarbeit nur koordiniere und nicht

12 Interview mit Carmel Borg am 22.9.2007.

13 Interview mit Andrew Fenek, Kommandeur der AFM und hauptverantwortlicher Kommandeur der Nautilus II-Missionen auf Malta im Jahre 2007. Das Interview fand am 5.10.2007 im Büro des Kommandeurs auf dem Hafengelände der Marineeinheit der AFM statt. 
für die Missionen verantwortlich sei. Alle Entscheidungsmacht liege bei den Mitgliedsländern. »It is a very difficult situation for Frontex. They do a good job, but they have limited resources. They have no vessels or aircraft. They can not order the tools. «14

Immer wieder unterstreicht Fenek, dass Frontex nur eine Mittleraufgabe übernehme, die EU-Mitgliedsländer müssten die Gerätschaften und Streitkräfte abstellen. Frontex, so Fenek, sei für den Frontex-Einsatz nicht verantwortlich, sondern die Mitgliedsländer: »The national officials are responsible for their crew at sea. ${ }^{15}$ Doch wie gestaltet sich die Zusammenarbeit, handelt es sich bei FrontexMissionen doch um eine gemeinsame europäische Unternehmung mit unterschiedlichen nationalen Rechtsgrundlagen. Fenek bemüht sich, die gemeinsame operative Entscheidungsfindung zu erläutern:

»I am the coordinator of the Nautilus mission. So we sit around the table in the coordination centre which is at Luqa and we discuss how we can do operations on a daily basis. We see how we can improve our operations; we see how we can better cover areas.

There is no rigid command structure. It's more like a committee where member states providing assets have one representative and we sit around the table and discuss how the operation is going. The last 24 hours, the reports we had, the next 24 hours we will plan in detail and we do a forecast for the next two or three days, when aircraft and vessels are going to go out and will be out at sea. It's on an ad hoc basis ... For example, the weather plays a big role in our decisions, the sea conditions ... $\ll^{16}$

Kommandeur Fenek beschreibt die Entscheidungsfindung an Land für die FrontexEinsätze auf See als eine Art ad hoc-Komitee der Sicherheitskräfte. Es nehmen ausschließlich Mitarbeiter der Sicherheitskräfte der Mitgliedsländer und FrontexBeamte an diesem Gremium teil. Gemeinsam werden so operative Entscheidungen getroffen, die ohne rechtliche Leitlinien und ungewisse rechtliche Basis über einen weiten Handlungsspielraum verfügen.

Dass tatsächlich lebensentscheidende Fragen zu den offenen Streitpunkten innerhalb des $a d$ hoc-Komitees der Sicherheitskräfte gehören, erzählt Kommandeur Fenek ebenfalls:

14 Interview mit Andrew Fenek am 5.10.2007.

15 Interview mit Andrew Fenek am 5.10.2007.

16 Interview mit Andrew Fenek am 5.10.2007. 
"We don't have major disagreements normally. We had problems when it came to considering actively diverting boats back. That's when we had major disagreements between us. I think it's all based on the effect that it is having on the security and stability of the country. If Malta is very affected by the situation then Malta is willing to take a tougher stand compared to a country that is not affected by the migration problem.

So you wanted to send boats back but other countries didn't agree?

I prefer not to go into this subject; it's a very sensitive subject. It never happened so I would prefer not to discuss it. «17

Fenek kommt hier auch auf eine Frage zu sprechen, die zentral für den FrontexEinsatz ist und auf europäischer Ebene, wie in den oben genannten Dokumenten gezeigt, umstritten bleibt. Leider ist er nicht bereit, weiter auf die Frage einzugehen, welche Länder zum Beispiel in welcher Weise auf See MigrantInnen zurück nach Libyen schieben wollen. Die Position Maltas, das Fenek zufolge sehr von Seemigration betroffen ist, wird jedoch deutlich: Auch in diesem Gremium vor Ort, das die Frontex-Einsätze bestimmt, ist Malta bereit, eine »tough position« für die Rückschiebung von Booten einzunehmen. Die »Frontstaatenperspektive« spielt dabei eine wichtige Rolle. Dass Malta dabei als Gastland der Mission einflussreicher ist als andere an Nautilus II beteiligte EU-Länder, beschreibt Kommandeur Borg ebenfalls: »Malta is the hosting nation, so we prepare the operational orders, the framework plan; we obviously host the ICC, which is the leading structure which is running such an operation.« Auch Sergio Carrera bestätigt am Beispiel der FrontexMission Hera und der Diskurse in Spanien, die ebenfalls einen Migrations-Notstand sehen, dass die »nationalen Arenen « (Carrera 2007: 12) eine zentrale Rolle in den Frontex-Einsätzen spielen. Andrew Neal (2009: 337) betont, dass die Wahrnehmung der Mittelmeermigration als Gefahr dabei je nach Geschichte der EU-Mitgliedsländer, der Rolle der Medien und der sozialen Lage der BürgerInnen stark variiere. Welchen Einfluss die Position Maltas innerhalb der Europäischen Union und der Frontex-Missionen entwickeln kann, wird im Folgenden deutlich.

Durch die Frontex-Missionen wird eine zentrale Annahme von Michael Lipsky (1980: XII) in besonderer Weise illustriert: Beamte und andere Vertreter des Staates beeinflussen nicht nur vor Ort den »open text « (Merry 1997: 30) der vorgegebenen Gesetze, sondern die Summe ihres Handelns und ihrer Entscheidungen machen im International Cooperation Centre (ICC) und auf See de facto die europäische Politik im Grenzraum Mittelmeer aus. Durch ihre zentrale Position im Grenzraum, ihren 
großen rechtlichen Spielraum, aber auch die Zwänge und Handlungslogiken, die sich in einer Runde ergeben, die ausschließlich aus Sicherheitskräften besteht, wird so vor Ort europäische Grenzpolitik bestimmt und Recht nicht nur implementiert, sondern auch geschaffen (Lipsky 1980: XV). Die Entscheidungen der Beamten haben dabei weitreichende Konsequenzen, besonders im Grenzraum, wo sie ein Informationsmonopol besitzen und ihre Handlungen kaum überprüft werden. Für MigrantInnen geht es dabei um Fragen, die über Leben oder Tod entscheiden können: Sollen sie nach Malta oder Libyen gebracht werden? Sind die MigrantInnen in Seenot oder nicht? Handelt es sich bei BootsmigrantInnen um »Illegale« oder Asylsuchende? »In street-level bureaucracies the objects of critical decisions - people actually change as a result of the decisions « (Lipsky 1980: 9; Hervorh. im Orig.). Diese Feststellung trifft auf See mit großer Folgenschwere zu.

\subsection{Europäische Flüchtlingsrechte auf See: Europäisierungsprozesse vor Ort}

Nach dem Gespräch mit Kommandeur Fenek bezüglich der Verantwortung für den Frontex-Einsatz und der Rechtsgrundlage für die Mission vor Ort bleiben einige Fragen offen. Vor allem die Rechtsgrundlage von Nautilus II ist unklar:

"Are you operating with the Schengen border codex?

Not yet. We are not part of Schengen yet.

But the other countries are ...

I don't know.

So on which legal basics are you operating? On which ground are the Frontex missions operating?

You have to ask Frontex that question. $^{18}$

Obwohl er versucht zu vermitteln, dass die Nautilus II-Mission mit ausreichender Sorgfalt und Vorbereitung durchgeführt wird, so wird doch klar, dass der Hauptkommandeur von Nautilus II nicht sicher ist, auf welchen rechtlichen Grundlagen seine Mission arbeitet. Der Schengener Grenzkodex, der laut EU-Recht auf alle Personen, die die Außengrenzen eines Mitgliedstaates überschreiten, Anwendung findet, ist es nicht, so Fenek. Malta war zum Zeitpunkt der Nautilus II-Mission noch nicht Mitglied des Schengener Abkommens. Da Fenek immer wieder betont hat, dass Frontex nicht für den Einsatz verantwortlich zeichnet, ist seine Aufforderung, doch Frontex und nicht ihn als hauptverantwortlichen Kommandeur nach der rechtlichen Basis des Einsatzes zu fragen, überraschend. Vor allem bleibt fraglich, auf

18 Interview mit Andrew Fenek am 5.10.2007. 
welcher Grundlage die ad-hoc-Einsatzgespräche der Sicherheitskräfte geführt werden, wenn die rechtliche Basis nicht klar ist. Welche Rechte werden dabei in Erwägung gezogen und welche nicht?

Auch AFM-Kommandeur Roger Gonzi bestätigt, dass es im Vorfeld der Mission nicht zu einer Einigung bezüglich der Vorgehensweise auf See kam:

»The problem was that every member state has a different interpretation of the law. So, there wasn't a common lowest denominator. So every nation kept commanding control over their own ships. And they act on national procedures. The procedures brought in by Frontex are very, very broad. [...] There is an operation order and when a ship meets a boat with migrants it will act according to national procedures. $\ll^{19}$

Als ich Gonzi frage, ob Fragen des Flüchtlingsrechts eine Rolle bei den Besprechungen im Vorfeld des Frontex-Einsatzes gespielt haben, stellt dieser klar: »No, no, that's not the case. $\ll^{20}$ Eher praktische Fragen, wie auf See vorzugehen sei und wie MigrantInnen nach Libyen zurückgebracht werden könnten, seien in diesem Gremium behandelt worden. Die unpräzisen rechtlichen Vorgaben von Frontex führen so dazu, dass in den entsprechenden Gremien, die für die praktische Durchführung von Frontex-Einsätzen zuständig sind, Flüchtlingsrechte keine Rolle spielen.

An den Aussagen des Kommandeurs zeigt sich, wie wichtig es ist, rechtliche Konstrukte, wie den auf nationaler, europäischer und internationaler Ebene verbrieften Flüchtlingsschutz, und die tatsächliche Anwendung des Rechts, die Reproduktion der Gesetzestexte in einem konkreten Raum zu einem konkreten Zeitpunkt, zu unterscheiden (F. v. Benda-Beckmann/ K. v. Benda-Beckmann 2007: 62). Diese Unterscheidung und die Rekonstruktion der Interaktionsketten lokaler, nationaler und transnationaler AkteurInnen (F. v. Benda-Beckmann et al. 2005: 19), die Rechte durch ihre Handlungen reproduzieren, modifizieren oder auch fallen lassen, geben nicht nur Aufschluss über Spannungsverhältnisse zwischen Rechtsanspruch und Rechtswirklichkeit. Sie sagen auch viel über den Wandel von Recht aus. In diesem Fall vor allem über Veränderungen und Entwicklungen in rechtspluralistisch geprägten transnationalen Rechtsprozessen wie der Europäisierung des EU-Rechts. Wiederum wird die Bedeutung der vor Ort agierenden Sicherheitskräfte in der Reproduktion von Recht dabei deutlich: Halten sie die Gesetzestexte des Flüchtlings-

19 Interview mit Roger Gonzi, Kommandeur der AFM, am 5.10.2007 im Hauptquartier der Armee (Luqua Barracks).

20 Interview mit Roger Gonzi am 5.10.2007. 
schutzes in ihren Gremien nicht für wichtig, dann gibt es in dem für die Wirksamkeit des Flüchtlingsschutzes entscheidenden Ort, dem Grenzraum, keine Flüchtlingsrechte.

Weitere zentrale Aspekte der komplexen rechtspluralistischen Situation ansprechend betont Gonzi auch, dass jedes Land auf See nach eigenen, unterschiedlichen Verfahrensmaßstäben agiert. Dass dies manchmal zu Konflikten führe, spricht Nachrichtenoffizier Sandro Grech an, da auch bei gemeinsamen Aktionen nach unterschiedlichen Standards vorgegangen werde. Dabei würden zum Beispiel maltesische Schiffe mit deutschen Hubschraubern kooperieren, aber - so Grech - »you have to remember that first of all you have your national interest. « ${ }^{21}$ Grechs Äußerung veranschaulicht den Umstand, dass, wie von Carrera (2007) und Neal (2009) beschrieben, in Europäisierungsprozessen von Recht weiter an lokalen oder nationalen Vorstellungen und Interessen festgehalten wird. Diese fließen, wie die Frontex-Mission illustriert, durch die unterschiedlichen Gremien und Gesprächsrunden in den Aushandlungsprozess um das neu entstehende europäische Recht ein. So ist eine Vielzahl an AkteurInnen, auch in informeller und zum Teil illegitimer Weise und auf verschiedenen Ebenen an der Schaffung von Recht beteiligt (vgl. F. v. Benda-Beckmann/K. v. Benda-Beckmann 2007: 65).

Dass durch die Aufrechterhaltung »nationaler Interessen« auch illegale Praktiken, zum Beispiel bedenkliche maltesische Vorgehensweisen bei der Seenotrettung von MigrantInnen, innerhalb der Frontex-Missionen angewendet werden, bestätigt Brian Vassallo, Direktor des UNHCR auf Malta: »What we have talked about before about Search and Rescue is done by the AFM. But it's done by the AFM within the context of the Frontex operation! «22

Es zeigt sich, dass die unter der Agentur Frontex vereinten Sicherheitskräfte ohne gemeinsame Leitlinien auf See zum Teil rechtlich nicht gedeckte und gegenläufige Vorgehensweisen praktizieren. Möglicherweise gefährliche Unsicherheiten für die BootsmigrantInnen können bei den operativen Vorgängen auf See damit Einzug halten. Im Rahmen der Europäisierung wird zum Teil auch gegen nationales und internationales Recht verstoßen. Ein besonders eklatantes Beispiel geht vor allem aus einem Vorfall hervor, den ich nun schildere.

21 Interview mit Sandro Grech, Mitarbeiter der maltesischen Polizei und im Rahmen von Nautilus II eingesetzt im nachrichtendienstlichen Bereich, am 12.10.2007 im Hauptquartier der AFM ( $L u$ qua Barracks).

22 Interview mit Brian Vassallo am 26.9.2007. Siehe zu Problemen bei der Rettung von BootsmigrantInnen wie der Verzögerung auch Klepp (2011 b). 


\subsection{Das Verschwinden der 700 - Rechtsunsicherheit und Intransparenz in der Frontex-Mission}

Zeigten die Gespräche mit den Kommandeuren menschen- und flüchtlingsrechtliche Schwachstellen im Hinblick auf die rechtliche Lage auf See und innerhalb der Frontex-Entscheidungsfindungsprozesse, so wurden diese Mängel bei Nachfragen bezüglich des Vorgehens der Mission auf See und der Frage, ob Nautilus II MigrantInnen nach Libyen zurückbringe und welche Rolle Libyen dabei spiele, noch deutlicher. Hier wurden das ganze Ausmaß der Rechtslücken und der Rechtskonfusion innerhalb der Frontex-Missionen und die Konsequenzen daraus für die BootsmigrantInnen und die Entwicklungen des europäischen Flüchtlingsschutzes deutlich.

Schon beim ersten Interview mit dem Sprecher des maltesischen Innenministeriums berichtete dieser, dass innerhalb einer gemeinsamen Rettungsaktion von Frontex und libyscher Marine MigrantInnen zurück nach Libyen gebracht worden seien. ${ }^{23}$ Einen Tag später wurde EU-Kommissar Frattini in einer Zeitungsmeldung folgendermaßen zitiert: »During the first phase (July) of Nautilus II, more than 700 illegal immigrants were intercepted and turned back towards their point of departure« (Camilleri 2007). Dies konnte nur bedeuten, dass Nautilus II regelmäßig nach Libyen zurückschiebt, da für den »point of departure« nur Libyen in Frage kam. Wenige Tage später folgte ein weiteres Interview im Innenministerium, wobei mir Mulvaney fast stolz erklärte, dass Libyen zwar nicht kooperiere, aber Frontex trotzdem MigrantInnen auf See zurück nach Libyen schicke. ${ }^{24}$

In den Interviews mit den Frontex-Kommandeuren zitierte ich die Aussage von Kommissar Frattini. Die Reaktionen der Kommandeure waren einerseits etwas verärgert, anderseits jedoch sehr einheitlich: Sie sagten genau das Gegenteil im Hinblick auf die Rückschiebungen von MigrantInnen nach Libyen, nämlich dass es keine Rückschiebungen gegeben habe. Kommandeur Borg bezüglich des FrattiniZitats:

$»$ Where does he get the information from? The fact is that turning back is not really on the cards.

So you don't think that 700 people were sent back?

I would prefer not to express an opinion on that point. [...] Maybe I don't know what Frattini says, I keep constant contact with Frontex ...« $\ll^{25}$

Interview mit Carmel Borg am 22.9.2007. 
Warum diese Art von Einsatz innerhalb von Nautilus II gar nicht möglich sei, präzisiert Gonzi:

"You should ask Frattini where he got it from. This is not true. Where the operation is done it's very far away from Libya, very far offshore. The closest to Libya is 60 nautical miles, this is $100 \mathrm{~km}$. We wanted it just in front of Libya, but it doesn't happen yet. For that we need Libyan co-operation. $\ll^{26}$

Eine Recherche im Internet und eine Rücksprache mit dem Autor des Artikels ergab keine Klarheit, ob Frattini tatsächlich das hier Zitierte gesagt hat. Nach einigen Rückfragen im Pressebüro von Frattini wurde mir mitgeteilt, dass Frattini falsch zitiert wurde und von $700 »$ Aufgegriffenen« gesprochen habe.

Mitten hinein in die gegenläufigen Informationen zum Verbleib der durch Frontex aufgegriffenen MigrantInnen auf See fällt ein Interview mit Vassallo, der von sich aus das Frattini-Zitat anspricht:

»Frattini said 700 people did not come to Malta.

And what does that mean?

That's what we are asking. We would not take the added step to say they were sent back. For obvious political reasons ... We won't say that, we'll stick to what has been said. We are asking: Where did they go? Where are they? What have they done to them? And we don't know. We don't even know what happened in the Frontex operation last year. We have no idea.

But this sounds crazy. 700 people disappeared?

I know. I agree with you. We have no idea where they are going. We have no clue. If they are going to Libya or to Italy. We have no idea.

Maybe they are brought to Italy?!

I don't know ... I don't know. The thing is, when we asked Frontex, we told them: Look, this might be happening and this and this and this, and we asked them, what do you think? They very clearly told us: Look, we have no say at all in the agreements the member states make amongst themselves on how to operate in Frontex operations. They told us that they come to Malta, they sit down; they say we have to work on Nautilus II for example and they discuss amongst themselves how it is going to work. Who will do what and how? [...] The Frontex issue is very dubious. It's very, very dubious. I can really say we are doing our best; we will hopefully get some information ...«27

27 Interview mit Brian Vassallo am 26.9.2007. 
Das Gespräch mit Vassallo bezüglich des Verbleibs von 700 durch Frontex aufgegriffene MigrantInnen zeigt, wie groß die Intransparenz und das Kompetenzwirrwarr rund um Nautilus II sind. Alles scheint hier innerhalb der Mission auf dem Meer möglich zu werden. Frontex ist nicht Willens oder in der Lage, der eigenen Partnerorganisation UNHCR ${ }^{28}$ Informationen über den Verbleib von 700 Menschen zu liefern.

Auch die Praktiken, die auf See im Umgang mit MigrantInnen angewendet werden, werden weder an die Öffentlichkeit noch an UNHCR weitergegeben: »What we know is very limited. [...] What we know is they are looking around for boats. We don't really know what they do when they see a boat, it's not very clear. We have asked Frontex, we have asked the Maltese to tell us what is happening. ${ }^{29}$ Eine Antwort habe UNHCR jedoch nicht erhalten, so Vassallo. Dies ist eine weitere Bestätigung des Informations- und Kontrollmonopols der Sicherheitskräfte im Grenzraum des Mittelmeers und der Vielfalt der Dinge, die auf See geschehen können. Sergio Carrera und Elspeth Guild (2010: 17) stellen fest, dass auch in späteren Frontex-Missionen die Einbeziehung von UNHCR, einem offiziellen Kooperationspartner der Agentur, nur unzureichend stattfindet. Damit kann die Intransparenz auf See nicht abgemildert werden, was eigentlich Ziel dieser Kooperation ist.

Der Journalist Roman Herzog veröffentlicht im Juni 2008 weitere Hinweise auf menschenrechtswidrige Praktiken innerhalb der Frontex-Einsätze. Ein an Nautilus beteiligter Einsatzleiter der italienischen Finanzpolizei (Guradia di Finanza) trifft dabei folgende Aussage:

»Als Italien sich mit einer Reihe weiterer Staaten an einen Tisch zusammensetzte, hat mich erstaunt, dass wir Italiener der illegalen Immigration rechtsstaatlich begegnen, indem wir zunächst Menschenleben retten und erst dann das Delikt ahnden. Andere Staaten benutzen den Begriff >diversion<, der bedeutet, die Menschen zu zwingen, nach Hause zurückzufahren. Es ist gar nicht so sehr die Tatsache, jemanden zur Umkehr zu zwingen, sondern wie man ihn dazu zwingt. Wir wurden bei offiziellen Treffen mit Einsatzplänen und schriftlichen Befehlen konfrontiert, laut denen die Abwehr der illegalen Einwanderer

28 UNHCR hat von Beginn an die Leitlinien-Gespräche für Frontex-Missionen in Brüssel mit einem eigenen Repräsentanten verfolgt. Am 23.6.2008 wurde in Warschau ein offizielles Abkommen zwischen UNHCR und Frontex unterzeichnet, das die »sehr unterschiedlichen Aufträge« der beiden Institutionen abstimmen soll: »The purpose and content of the cooperation reflected in these Working Arrangements constitute a fair balance between the very different remits of Frontex and UNHCR and a common goal to promote an efficient EU integrated border management system, which is fully compliant with human rights (Frontex 2008).

Interview mit Brian Vassallo am 26.9.2007. 
darin besteht, an Bord der Schiffe zu gehen und die Lebensmittel und den Treibstoff von Bord zu entnehmen, so dass die Immigranten dann entweder unter diesen Bedingungen weiterfahren können oder aber lieber umkehren« (Herzog 2008).

Der Kommandeur bekräftigt seine Aussage nochmals und verabschiedet sich mit den Worten: »Über das, was wirklich auf dem Meer geschieht, gibt es keine direkten Informationen, und es wird auch niemals welche geben« (Herzog 2008).

Zum einen gibt es zu wenige Auskünfte über die Geschehnisse auf See innerhalb der Frontex-Missionen. Zum anderen sind jedoch auch Kompetenzen und Verantwortlichkeiten bei den Einsätzen auf See und für die Menschen, die dort aufgegriffen werden, unklar. Frontex-Direktor Ilka Laitinen verweist in Interviews auf die Verantwortlichkeit der Mitgliedsländer (Herzog 2008). Diese delegieren wiederum immer wieder an die Agentur Frontex, also den relativ kleinen Verwaltungsapparat in Warschau. Im Rahmen des Verantwortungsvakuums zwischen Frontex und den Mitgliedsländern scheinen sich dabei auf dem Mittelmeer schwere Menschenrechtsverletzungen abzuspielen. Es zeigt sich, dass die direkten Mitarbeiter der Agentur innerhalb der Missionen nicht als Kontrollgremium fungieren und auch UNHCR ausgeschlossen bleibt. Die Koordinationsaufgabe von Frontex ist bei so viel Nicht-Wissen bezüglich von Informationen, welche die Mission direkt betreffen, fraglich. Da Frontex jedoch die Rahmenbedingungen und die Ziele der Mission bestimmt, reicht ein Verweis auf die Mitgliedsländer nicht aus, um sich als Agentur aus der Verantwortung zu nehmen. Die Agentur und ihre Mitarbeiter müssen eine Entscheidung über ihre Rolle im Rahmen der Missionen an den EU-Grenzen herbeiführen und es muss eine klare Kompetenzverteilung zwischen nationaler und supranationaler Ebene erkennbar werden.

\subsection{Das Puzzle Nautilus II setzt sich zusammen}

Ob es innerhalb der Nautilus II-Mission Kooperationen mit Libyen gab und ob tatsächlich MigrantInnen nach Libyen zurückgeschoben wurden, lässt sich aufgrund der gegenläufigen Informationen nicht abschließend beurteilen. Durch meine immer wieder gestellten Fragen, wo genau Nautilus II patrouilliere, wie Nautilus II auf See mit aufgebrachten MigrantInnenbooten umgehe und wohin die MigrantInnen gebracht werden, wenn nicht nach Libyen, setzt sich das Puzzle »Nautilus II auf See« dennoch zusammen. Auch wenn die letztendliche Verlässlichkeit der von den Kommandeuren gegebenen Informationen schwierig zu beurteilen ist, so gab es doch bezüglich einiger Fragen einhellige Aussagen: Im Gegensatz zu nationalen maltesischen Einheiten patrouilliert Nautilus II nicht nur in maltesischen Gewässern und 
maltesischem SAR-Gebiet ${ }^{30}$, sondern auch in der libyschen SAR-Zone. Ausgespart bleiben jedoch, solange Libyen nicht offiziell kooperiert, libysche Hoheitsgewässer. Bezüglich der aufgegriffenen MigrantInnen erklären alle Kommandeure, dass innerhalb von Nautilus II die MigrantInnen zwischen Malta und Italien verteilt würden.

Grech erklärt, dass das Operationsgebiet in drei Bereiche aufgeteilt ist: »Two areas go to Italy, one goes to Malta. $\ll^{31}$ Dennoch bleibt die Aufteilung der MigrantInnen ein Problem, wie die Anlaufschwierigkeiten von Nautilus III gezeigt haben. »It is always an issue ...«, wie es Gonzi formuliert und damit wiederum Kompetenzlücken und den Handlungsspielraum der AkteurInnen vor Ort deutlich macht.

Angesprochen auf die Aufgabe der Mission und ihre Möglichkeiten auf See, solange Libyen nicht kooperiere, herrscht Einigkeit unter den Kommandeuren. Sie sei sehr begrenzt und grundsätzlich nur im Monitoring-Bereich zu sehen, so Kommandeur Borg: »First of all, we have to rise the awareness of how things are happening, there is an intelligence aspect. Before you can stop a flow you have to be aware of what is happening. $\ll^{32}$ Die Experten der verschiedenen Mitgliedsländer und Grech hätten die Aufgabe, die MigrantInnen an Land zu interviewen, um mehr über die Organisation ihrer Reisen und ihre Reisewege zu erfahren. Ansonsten laufe ein maltesischer Einsatz innerhalb einer Frontex-Mission nicht anders ab, als bei einer nationalen Aktion. Man rede mit den MigrantInnen und versuche herauszufinden, was sie vorhaben; meistens wollten sie nach Italien. »When we see that they are safe, they continue with their journey «33, so Kommandeur Fenek. Innerhalb der Frontex-Mission Nautilus II, Phase II wurden laut Fenek zwischen 1500 und 2000 MigrantInnen in dieser Weise aufgebracht.

Die unterschiedlichen Interpretationen, wann ein Boot sicher ist und gerettet werden muss, und wann nicht, bleiben auch innerhalb des Frontex-Einsatzes bestehen, wie Gonzi nochmals bestätigt:

»There is an operation order and when a ship meets a boat with migrants it will act according to national procedures. Italy's interpretation is that when they find a boat they work according to a certain procedure. That is how they do it. If we think it's different we do our own. ${ }^{34}$

30 »SAR « steht für »Search and Rescue«.

31 Interview mit Sandro Grech am 12.10.2007.

32 Interview mit Carmel Borg am 22.9.2007.

33 Interview mit Andrew Fenek am 5.10.2007.

34 Interview mit Roger Gonzi am 5.10.2007. 
Alle Kommandeure sind sich einig, dass für ein erfolgreiches Arbeiten der Nautilus-Missionen eine enge Kooperation mit Libyen unabdingbar sei: »This ist the crux of it all. We could have all assets in the world, but as long as Libya doesn't cooperate ... «35 Auch dass es diese Kooperation in der Zukunft geben wird, wahrscheinlich auch in libyschen Gewässern und auf libyschem Territorium, ist für alle Kommandeure klar.

Mit der heutigen Perspektive auf die Kooperation zwischen Libyen und der EU haben die Kommandeure der AFM Recht behalten. So gibt es auch nach dem Fall des Gaddafi-Regimes eine enge Zusammenarbeit mit Libyen, obwohl die Menschenrechtssituation für Menschen aus Subsahara-Afrika schlecht bleibt (Médecins sans Frontières 2012). Eine Presseerklärung des Rats vom 31.1.2013 beschreibt die weitgehende Kooperation mit Libyen im Bereich der Grenzsicherung. Europäische Sicherheitskräfte sollen dabei Trainings- und Monitoringaufgaben übernehmen, aber auch kurzfristig sicherstellen, dass die Grenzen innerhalb Libyens besser geschützt werden, so heißt es vage (Council of the European Union 2013).

Insgesamt beurteilen die Kommandeure und der Nachrichtenoffizier die FrontexMissionen sehr positiv. Die Atmosphäre unter den Sicherheitskräften der verschiedenen Mitgliedsländer sei sehr gut, »a nice multicultural atmosphere«, wie Grech es formuliert. Alle sind sich einig, dass die Zukunft der Sicherung der EU-Außengrenzen bei Frontex liegt. Die Unsicherheiten und Schwierigkeiten, die es jetzt gebe seien höchstens »teething problems", wie es Grech nennt. »Things that come up at work. You weren't prepared to it and then it comes up. ${ }^{36}$ Dass sich im Zuge dieser »teething problems « und im Umfeld der rechtlichen Unsicherheiten rund um Frontex Verantwortlichkeiten im Flüchtlingsschutzbereich verflüchtigen und informelle und illegale Praktiken der nationalen Sicherheitsverbände auf der operativen Ebene europäisiert und verstetigt werden, wird nun weiterführend diskutiert.

\section{Frontex auf See und die Europäisierung des Flüchtlingsrechts}

In diesem Abschnitt werden einige Folgen und Aspekte der Europäisierung des europäischen Flüchtlingsschutzes diskutiert. Zunächst geht es um Verantwortlichkeiten im Flüchtlingsschutz, die im Europäisierungsprozess Gefahr laufen, zwischen der Ebene der Mitgliedsländer und der EU-Ebene in einer Weise verlagert zu werden, die Unklarheiten schafft. Zuständigkeiten, wie zum Beispiel für BootsmigrantInnen auf See und ihre Asylgesuche, werden somit unkenntlich. Malta und andere Grenzländer scheinen zudem eine wichtige Rolle in den Frontex-Leitlinien-

35 Interview mit Carmel Borg am 22.9.2007.

36 Interview mit Sandro Grech am 12.10.2007. 
Gesprächen in Brüssel zu spielen. Sie beeinflussen damit maßgeblich die Zukunft des europäischen Flüchtlingsschutzes im Grenzraum. Es zeigt sich, dass kleine Länder wie Malta dabei im Europäisierungsprozess an Einfluss gewinnen können.

\subsection{Die Verflüchtigung von Verantwortung im europäischen Flüchtlingsschutz}

Durch Europäisierungsprozesse, die eigentlich mehr Homogenität und ein harmonisiertes Flüchtlingsrecht schaffen sollten, ist es innerhalb der EU zu Widersprüchen, Unsicherheiten und Fragmentierungen gekommen, die viel Spielraum für Interpretationen und Interessenspolitik im EU-Flüchtlingsschutz lassen (F. v. BendaBeckmann et al. 2005: 19). Dieser Raum wird von AkteurInnen unterschiedlicher Ebenen genutzt, um Recht gemäß eigener Interessen zu formen oder auch zu ignorieren. Verantwortlichkeiten, die auf nationaler Ebene klar sind, können sich im Zuge der Europäisierung verschieben und verwischen oder sogar aufheben, wie die Frontex-Missionen auf See zeigen: »In many areas of policy making it is increasingly difficult to see where national competence ends and EU competence begins « (Shore 2006: 720). So werden Menschrechtsverletzungen im Grenzraum gefördert und ihre Aufklärung und Strafverfolgung nahezu unmöglich.

Innerhalb der Frontex-Missionen auf See werden offizielle zivile Partnerorganisationen wie UNHCR außen vor gelassen. Zudem wird in den undurchschaubaren Befehlsstrukturen und Kommandoketten Verantwortung aufgeteilt oder weitergeben, ohne entsprechende Verfahren und Mechanismen von transnationaler Verantwortung gefunden zu haben. Die Mitgliedstaaten und ihre Hoheitsträger, die Kommandanten, "verstecken « sich in dieser Weise hinter der europäischen Agentur Frontex, die nicht mit souveränen Rechten ausgestattet ist. Verantwortlichkeiten gehen dabei zum Teil verloren (F. v. Benda-Beckmann et al. 2009: 14). Außerdem werden zentrale politische Fragen, wie die Verfahrensweise mit BootsmigrantInnen auf See und die Kompetenzverteilungen innerhalb der Frontex-Missionen »entpolitisiert « und der inadäquaten Ebene von ad hoc-Gesprächsrunden von Beamten und Sicherheitskräften überlassen (F. v. Benda-Beckmann et al. 2009: 15). Durch die Frontex-Risikoanalysen und die Erhebung von Wissen zur Organisation von Migration durch Experten innerhalb von Frontex werden zudem die Bedingungen für einen »gouvernementalen Zugriff auf die migrantische Bevölkerung der Grenze« (Kasparek 2010: 115) erst geschaffen. Welche negativen Folgen eine in dieser Weise vor sich gehende Europäisierung für den Schutz der Menschenrechte auf dem Mittelmeer und für den Flüchtlingsschutz haben könnte, zeigen die Ignoranz gegenüber Flüchtlingsrecht in den Frontex-Foren, die Intransparenz der Geschehnisse 
auf See sowie der ständige Streit, wohin aufgegriffene BootsmigrantInnen gebracht werden sollen und wer für sie verantwortlich ist. Sarah Leonard sieht als Grund für die fehlende Transparenz der Arbeit von Frontex vor allem das Vorgehen während der Schaffung der Agentur: Da das EU-Parlament als traditionell starker Verfechter der Menschenrechte zu wenig in die Verabschiedung der Frontex-VO und in die Kontrolle von Frontex einbezogen sei, gebe es große Lücken in Fragen der Transparenz und Verantwortlichkeit (Leonard 2009: 385).

\section{2 »We are standing up there« - Die Leitlinien-Gespräche auf europäischer Ebene}

Bezüglich der Zukunft der Frontex-Einsätze und den weiteren Entwicklungen auf See auch im flüchtlingsrechtlichen Bereich bin ich abschließend natürlich daran interessiert, von Kommandeur Borg Informationen zu den Leitlinien-Gesprächen in Brüssel zu erhalten, die diese Zukunft bestimmen werden. Die Leitlinien werden in einer Gruppe von Repräsentanten der einzelnen Länder in Brüssel verhandelt, wie Borg beschreibt. Die Einigungsgespräche seien noch in einer recht frühen Phase, es würde jedoch unter Hochdruck gearbeitet, da, wie Borg es formuliert, dringend praktische Leitlinien »an der Frontlinie « ${ }^{37}$ für die Frontex-Einsätze gebraucht werden, um den Kommandeuren der Schiffe mehr Rechtssicherheit zu geben. Sie seien es, die letztlich die Verantwortung auf See trügen. Da die nationalen Standpunkte zum Teil sehr unterschiedlich seien, gebe es wichtige Punkte zu klären. Ein Hauptstreitpunkt sei auch auf dieser Ebene, welches am Einsatz beteiligte Land für die Aufnahme der aufgegriffenen Flüchtlinge zuständig ist:

\section{»Is this a big issue?}

Of course it is a big issue! For Nautilus the german government made it very clear. Everyone rescued by a german helicopter will be taken to Germany. [...] The helicopter is sovereign territory so that was their first entry. But what about when you are taking on board 250 people ... It's all up to discussion. There are some legal authorities who say even when you are alongside the ship they can claim asylum. This is another reason why we do transfers with small boats Why? 
Because there are some legal authorities who say when you bring them alongside your warship they can claim asylum, you know. These are things that have to be handled. « ${ }^{38}$

Ein Hauptproblem der Nautilus II-Mission auf rechtlicher Ebene wird hier nochmals deutlich: Wer ist für innerhalb der gemeinsamen Mission aufgegriffene Flüchtlinge zuständig? An dieser Streitfrage, die auch eine starke politische Dimension hat, und unter anderem die viel beschworene Solidarität der EU-Mitgliedsländer mit den Ländern an der EU-Außengrenze betrifft, scheiterte auch der pünktliche Start der Nautilus III-Mission im Jahre 2008. Malta und Italien hatten sich geweigert, weiterhin den überwiegenden Teil der MigrantInnen aufzunehmen, die von der Mission aufgegriffen werden (Frontex 2008). Erst mit einer Verzögerung von mehreren Wochen wurde Nautilus III im Mai 2008 gestartet.

Im Zusammenhang mit der Verantwortung für aufgegriffene MigrantInnen und ihr potentielles Asylgesuch ist ein weiterer Aspekt, den Kommandeur Borg anspricht, die Strategie Maltas, sich den MigrantInnen mit kleinen Booten zu nähern, um dem Zusammentreffen einen informelleren Charakter zu verleihen und das Recht auf Zugang zu Asyl zu vermeiden. Er beschreibt damit einen Versuch der maltesischen Armee, ihren Verpflichtungen gegenüber Asylsuchenden durch rechtliche Winkelzüge zu entgehen. Die rechtliche Unsicherheit rund um die Mission ist, wie schon diskutiert, die Ursache dafür, dass diese Formen des Lavierens auch innerhalb von Frontex-Einsätzen möglich ist. Eigentlich ergeben sich in dieser Situation die Verpflichtungen der Sicherheitskräfte laut UNHCR und entsprechender Rechtsgutachten daraus, dass der Staat bei der »Ausübung von Hoheitsgewalt, [...] die völkerrechtlichen Menschen- und Flüchtlingsrechte beachten muss « (FischerLescano/Löhr 2007: 22; siehe auch Weinzierl/Lisson 2007: 16). Es ist dabei nicht von Bedeutung, welche Größe das Schiff hat, mit dem die BootsmigrantInnen aufgegriffen werden.

Kommandeur Borg erklärt mir weiter, dass die unterschiedlichen Praktiken der Mitgliedsländer auf See, die schon beim Thema Seerettung von MigrantInnen eine wichtige Rolle gespielt haben, auch Hauptgegenstand der Leitlinien-Gespräche seien: »So for example, if a boat is overloaded, is it in distress or not? That should come out of the guidelines. ${ }^{39}$ Der vorherige Abschnitt hat dabei gezeigt, dass schon bei dieser wichtigen Frage die Meinungen der maltesischen und der italienischen Sicherheitskräfte weit auseinander liegen und dass auch hier die Feststellung von 
Carrera (2007) und Neal (2009), dass die nationalstaatlichen Prägungen das Vorgehen der Sicherheitskräfte entscheidend prägen, zutrifft.

Eine Frage, die ich Kommandeur Borg bezüglich der Leitlinien-Gespräche stelle, zielt außerdem auf die Rolle Maltas innerhalb dieses europäischen Forums ab:

\section{"Which role plays Malta in the guideline talks?}

It's a very coactive role. We are in an exposed position so it is our interest to do so. The positive thing is that is a one country - one voice system. So Malta is as important as Germany. And we are standing up there, because if you don't stand up they will pass you the entire burden. So we stand up and talk. The countries that have a very coactive role are Italy, Greece, Cyprus and Malta. Not Spain, although I thought so, but they are not so active. So all the countries that have direct experience with illegal migration .... $\ll^{40}$

Hier wird auf der Ebene der Leitlinien-Gespräche für die Frontex-Einsätze deutlich, dass Malta wiederum eine besonders starke Rolle innerhalb der EU-Foren einnimmt, die sich mit dem Umgang mit MigrantInnen auf See beschäftigen. Gemeinsam mit anderen Staaten, die vermutlich eine ähnliche »Frontstaatenperspektive« wie Malta einnehmen, dominiert Malta die Gespräche. Zugute kommt kleinen Ländern wie Malta, dass sie dabei dasselbe Stimmrecht haben wie Länder mit einer weit höheren Einwohnerzahl. Welche Position Malta dabei bezüglich des Non-Refoulement-Gebotes einnimmt und welchen Stellenwert Malta flüchtlingsrechtlichen Maßstäben auf See einräumt, wurde dabei in einem Interview mit St. John klar:

»Our view is: They have come to us from Libya, but this is not where they are originated from. The place that one would turn back to is Eritrea or Somalia. But we are not turning them back to Eritrea or Somalia; we are turning them back to Libya.

But Libya has no asylum system. This is really a discussed point, no?

I think in fine legalistic terms that is absolutely true, but we have to deal with the reality. And the reality on the ground is that although Libya is not a signatory to this agreement it's got its own laws which they tell us take care of the illegal immigrants that are in their country. (Pause) And it's really not for us to second guess what the Libyans do ... « ${ }^{41}$

Der Berater der Regierung nennt den Grundpfeiler des internationalen Flüchtlingsschutzsystems, das Gebot des Non-Refoulement, ein »juristisches Detail«, auf das

41 Interview mit Jason St. John am 2.10.2007. 
Malta keine Rücksicht nehmen könne. Wie Kommandeur Borg spricht zudem auch St. John an, dass dem großen Einfluss der kleinen Insel Malta auch das EU-Abstimmungssystem innerhalb vieler europäischer Foren zugutekäme, das jedem Land, egal welcher Größe und Einwohnerzahl, eine Stimme zurechnet: »It's one of the benefits of the EU«, so der Berater der Regierung bezüglich der LeitlinienGespräche. »Malta is the leading voice ... My first advice in the report was that irregular immigration was the prime foreign policy issue that Malta had to deal with and that secondly they had a voice and they had to make it heard. $\aleph^{42}$

\section{Ausblick}

Es zeigt sich, dass die »Frontstaatenperspektive« der Grenzländer im Mittelmeer im Bereich des Flüchtlingsschutzes und der Kontrolle der EU-Außengrenzen eine einflussreiche Position innerhalb der europäischen Institutionen einnimmt. Grenzländer wie Malta dominieren die Leitlinien-Gespräche und andere Foren, in denen das Vorgehen an den Außengrenzen der EU und damit die Zukunft des europäischen Flüchtlingsschutzes diskutiert wird. Die vermeintlich peripher gelegenen Länder der Europäischen Union werden für die Entwicklungen innerhalb des EU-Flüchtlingsschutzes zu einem machtvollen Akteur. Der dialektische Prozess, der sich zwischen angenommenem Macht-Zentrum und vermeintlicher Peripherie der Europäischen Union im Bereich Flüchtlingsschutz entwickelt hat, zeigt, dass die formellen Regeln der EU teilweise umgekehrt werden können. In den Ländern an den EUAußengrenzen wird europäisches Recht auch in der Implementierungsphase nicht einfach umgesetzt, sondern die oft großen Spielräume des EU-Rechts werden genutzt und nach lokaler und nationaler Interessenlage modifiziert, interpretiert und weiter beeinflusst. Die Europäisierung von Recht geht somit nicht immer mit einer viel zitierten Einschränkung der nationalstaatlichen Souveränität und Handlungsmacht einher: Es zeigt sich vielmehr, dass innerhalb der EU kleine Staaten an Einfluss über ungleich größere Länder gewinnen und ihre Handlungsmacht ausbauen können.

Das Gebot des Non-Refoulement und weitere Aspekte, die für ein funktionierendes Flüchtlingsschutzsystem im Grenzraum und damit innerhalb der gesamten EU entscheidend sind, befinden sich inmitten einer Aushandlungsphase, die geprägt ist von rechtlichen Erschütterungen und Unsicherheit. Wie die Studie an der Grenze zeigt, sind die ausschlaggebenden AkteurInnen in dieser »multi-sited arena of negotiation« (F. v. Benda-Beckmann et al. 2005: 19) nicht die demokratisch legiti- 
mierten VertreterInnen der europäischen Zivilgesellschaften in offiziellen EU-Entscheidungsgremien. Es sind vielmehr die SicherheitsexpertInnen und Militärangehörigen, die vor Ort Recht interpretieren, es umsetzen (oder auch nicht) und somit zukünftige Entwicklungen im europäischen Flüchtlingsschutz beeinflussen. Das Gebot des Non-Refoulement auf See wurde im Gerichtsverfahren »Hirsi Jamaa und andere gegen Italien« im Februar 2012 durch den Europäischen Gerichtshof für Menschenrechte und durch die Änderung der Frontex-VO vom 13. September 2011 formal gestärkt. In der veränderten Version heißt es nun:

»Die Agentur erfüllt ihre Aufgaben unter umfassender Einhaltung der einschlägigen Rechtsvorschriften der Union, einschließlich der Charta der Grundrechte der Europäischen Union, dem einschlägigen Völkerrecht, einschließlich des am 28. Juli 1951 in Genf geschlossenen Abkommens über die Rechtsstellung von Flüchtlingen (»Genfer Konvention«), der Verpflichtungen im Zusammenhang mit dem Zugang zu internationalem Schutz, insbesondere des Grundsatzes der Nichtzurückweisung, sowie der Grundrechte, wobei die Berichte des in Artikel 26 a genannten Konsultationsforums zu berücksichtigen sind« (Europäisches Parlament 2011).

Die Anwendung des Rechts liegt jedoch weiter in den Händen der Sicherheitskräfte im intransparenten Grenzraum, wo die Umsetzung und somit das »lebendige« Recht schlecht einzuklagen und durch die Zivilgesellschaft zu kontrollieren sind. UNHCR hat weiterhin keinen direkten Zugang zur Arbeit der Missionen (Carrera/Guild 2010: 15). Verletzungen der Menschen- und der Flüchtlingsrechte innerhalb der Missionen sind weiter festzustellen (Carrera/Guild 2010). Zudem ist, wie das Beispiel Malta zeigt, das politische Klima besonders wichtig für die Umsetzung von Flüchtlingsrechten. Dieses scheint sich für Flüchtlinge in den Ländern der EU-Außengrenzen (vgl. Klepp 2011 a) und auch in den restlichen Ländern Europas in den letzten Jahren nicht zu verbessern. Das Gebot des Non-Refoulement und andere wichtige Aspekte des EU-Flüchtlingsrechts sind deshalb in den Grenzgebieten der Europäischen Union weiter in Gefahr und könnten von dort schrittweise modifiziert und auch abgebaut werden. Die im Artikel beschriebenen Phänomene können somit auch nicht als »teething problems « der ersten Jahre der Frontex-Agentur beschrieben werden. Die Ursachen für die Differenz zwischen Rechtsanspruch und Rechtswirklichkeit an den EU-Außengrenzen sind wesentlich komplexer, wie der Artikel gezeigt hat. Dabei tragen alle Beteiligten an den Frontex-Missionen die volle Verantwortung für die Umsetzung des Flüchtlingsschutzes und die Einhaltung des Gebots des Non-Refoulement (Carrera/Guild 2010: 17). Ansonsten drohe, wie Carrera und Guild feststellen, dass »the EU will be shamed before the whole of the inter- 
national community « (Carrera/Guild 2010: 17). Die EU riskiert somit mit den Frontex-Einsätzen einerseits ihre Glaubwürdigkeit nach außen, anderseits jedoch auch ihr eigenes starkes Fundament: die Menschenrechte und deren rechtsstaatliche Umsetzung.

\section{Literatur}

Benda-Beckmann, Franz von/Benda-Beckmann, Keebet von 2007: Transnationalisation of Law, Globalisation and Legal Pluralism: A Legal Anthropological Perspective, in: Antons, Christoph/Gessner, Volkmar (Hrsg.): Globalisation and Resistance: Law Reform in Asia since the Crisis, Oxford, 53-80.

Benda-Beckmann, Franz von/Benda-Beckmann, Keebet von/Eckert, Julia 2009: Rules of Law and Laws of Ruling: Law and Governance between Past and Future, in: dies. (Hrsg.): Rules of Law and Laws of Ruling: On the Governance of Law, Farnham, 1-30.

Benda-Beckmann, Franz von/Benda-Beckmann, Keebet von/Griffiths, Anne 2005: Mobile People, Mobile Law: An Introduction, in: dies. (Hrsg.): Mobile People, Mobile Law: Expanding Legal Relations in a Contracting World, Aldershot, $1-25$.

Bourdieu, Pierre 1997: Das Elend der Welt, Konstanz.

Camilleri, Ivan 2007: Patrols Halt 700 Migrants, in: The Times of Malta, 19.9.2007, in: http://www.timesofmalta.com/articles/view/20070919/local/patrols-halt-70 0 -migrants.4640; 15.3.2013.

Carrera, Sergio 2007: The EU Border Management Strategy. FRONTEX and the Challenges of Irregular Immigration in the Canary Islands (CEPS Working Document, No. 261/March 2007), in: http://aei.pitt.edu/7385/; 9.4.2013.

Carrera, Sergio/Guild, Elspeth 2010: >Joint Operation RABIT 2010<-FRONTEX Assistance to Greece's Border with Turkey: Revealing the Deficiencies of Europe's Dublin Asylum System (CEPS Papers in Liberty and Security in Europe, November 2010), in: http://www.ceps.eu/book/\%E2\%80\%98joint-oper ation-rabit-2010\%E2\%80\%99-\%E2\%80\%93-frontex-assistance-greece $\% \mathrm{E} 2 \%$ 80\%99s-border-turkey-revealing-deficiencies; 9.4.2013.

Council of Europe 2013: EU Prepares Support to Border Management in Libya (5823/13 Presse 37, Brussels, 31 January 2013), in: http://www.consilium.europ a.eu/uedocs/cms data/docs/pressdata/EN/foraff/135129.pdf; 17.3.2013.

Europäisches Parlament 2011: Legislative Entschließung des Europäischen Parlaments vom 13. September 2011 zu dem Vorschlag für eine Verordnung des Europäischen Parlaments und des Rates zur Änderung der Verordnung (EG) 
Nr. 2007/2004 des Rates zur Errichtung einer Europäischen Agentur für die operative Zusammenarbeit an den Außengrenzen der Mitgliedstaaten der Europäischen Union (FRONTEX), KOM (2010) 0061 - C7-0045/2010 - 2010/0039 (COD), in: http://eur-lex.europa.eu/LexUriServ/LexUriServ.do?uri=OJ:C:2013 :051E:0157:0158:DE:PDF; 8.4.2013.

European Council on Refugees and Exiles 2007: Defending Refugees' Access to Protection in Europe, in: http://www.ecre.org/resources/policy_papers/988; 20.1.2012.

Fischer-Lescano, Andreas/Löhr, Tillmann 2007: Rechtsgutachten. Menschen- und flüchtlingsrechtliche Anforderungen an Maßnahmen der Grenzkontrolle auf See, Berlin.

Fischer-Lescano, Andreas/Tohidipur, Timo 2007: Die europäische Grenzschutzagentur FRONTEX, in: ASYLMAGAZIN 5/2007, 19-28.

Frontex 2006: Allgemeiner Tätigkeitsbericht für 2005, in: http://www.frontex.eu ropa.eu/gfx/frontex-/files/justyna/microsoft_word_-_frontex-2008-0003-00-00 -ende.pdf; 10.5.2011.

Frontex 2007 a: Frontex-Jahresbericht 2006, in: http://www.frontex.europa.eu/gf $\mathrm{x} /$ frontex/-files/justyna/microsoft_word_-_frontex-2008-0001-00-00-ende.pdf; 12.5.2011.

Frontex 2007 b: Examples of Accomplished Operations, in: http://www.frontex. europa.eu/examples-_of_accomplished_operati/art6.html; 25.6.2010.

Frontex 2008: News Release. 7.5.2008. Go ahead for Nautilus 2008, in: http:// www.frontex.europa.eu/newsroom/news_releases/art36.html; 10.10.2011.

Frontex 2013: Press Releases, in: http://www.frontex.europa.eu/media-centre/press -releases; 21.2.2013.

Herzog, Roman 2008: Krieg im Mittelmeer. Von der Cap Anamur zu Frontex und Europas neuen Lagern (Skript zum Radiofeature, gesendet am 22.6.2008 auf NDR Info, am 26.6.2008 auf SWR2), in: http://www.swr.de/swr2/programm/ sendungen/feature/-/id=659934/nid=659934/did=3510294/w8hs6t/index.html; 17.3.2013.

Jeandesboz, Julien 2008: Reinforcing the Surveillance of EU Borders. The Future Development of FRONTEX and EUROSUR (CEPS CHALLENGE Programme Research Paper, No. 11/August 2008), in: http://aei.pitt.edu/9402/; 9.4.2013. Kasparek, Bernd 2010: Laboratorium, Think Tank, Doing Border: Die Grenzschutzagentur Frontex, in: Hess, Sabine/Kasparek, Bernd (Hrsg.): Grenzregime. Diskurse, Praktiken, Institutionen in Europa, Berlin, 111-127.

Kasparek, Bernd/Marischka, Christoph 2008: Glossar, in: Pflüger, Tobias (Hrsg.): Aufgaben und Strukturen der Europäischen Agentur für die operative Zusam- 
menarbeit an den Außengrenzen (Materialien gegen Krieg, Repression und für andere Verhältnisse, Nr. 4), 42-50.

Klepp, Silja 2007: Ankunft und Aufnahme von Flüchtlingen in Italien. Eine ethnographische Reise an die Grenzen Europas, Saarbrücken.

Klepp, Silja 2011 a: Europa zwischen Grenzkontrolle und Flüchtlingsschutz. Eine Ethnographie der Seegrenze auf dem Mittelmeer, Bielefeld.

Klepp, Silja 2011 b: A Double Bind: Malta and the Rescue of Unwanted Migrants at Sea. A Legal Anthropological Perspective on the Humanitarian Law of the Sea, in: International Journal of Refugee Law 23: 3, 538-557.

KOM - Kommission der Europäischen Gemeinschaften 2006: Mitteilung der Kommission an den Rat. Ausbau von Grenzschutz und -verwaltung an den südlichen Seegrenzen der Europäischen Union, KOM (2006) 733 endgültig vom 30.11.2006, in: http://eur-lex.europa.eu/LexUriServ/LexUriServ.do?uri=COM: 2006:0733:FIN:DE:PDF; 8.4.2013.

KOM - Kommission der Europäischen Gemeinschaften 2008: Mitteilung der Kommission an das Europäische Parlament, den Rat, den europäischen Wirtschaftsund Sozialausschuss und den Ausschuss der Regionen. Prüfung der Schaffung eines Europäischen Grenzkontrollsystems (EUROSUR), KOM (2008) 68 endgültig vom 13.2.2008, in: http://eur-lex.europa.eu/LexUriServ/LexUriServ.do? uri=COM:2008:0068:FIN:DE:PDF; 8.4.2013.

Lavenex, Sandra/Ucarer, Emek M. 2004: The External Dimension of Europeanization: The Case of Immigration Policies, in: Cooperation and Conflict 39: 4, 417-443.

Leonard, Sarah 2009: The Creation of FRONTEX and the Politics of Institutionalisation in the EU External Borders Policy, in: Journal of Contemporary European Research 5: 3, 371-388.

Lipsky, Michael 1980: Street-Level Bureaucracy. Dilemmas of the Individual in Public Services, New York, NY.

Mèdecins sans Frontières 2012: Libya: Detainees Tortured and Denied Medical Care. MSF Suspends Work in Detention Centers in Misrata, in: http://www.doc torswithoutborders.org/press/release.cfm?id=5744\&cat=press-release\&ref=ne ws-index; 22.2.2013.

Merry, Sally E. 1997: Legal Pluralism and Transnational Culture: The Kaho'okolokolonui Kanaka Maoli Tibunal, Hawai'i, 1993, in: Wilson, Richard A. (Hrsg.): Human Rights, Culture and Context. Anthropological Perspectives, London, 28-48.

Micaleff, Mariella 2006: One Way Ticket: The Illegal Immigrant in Malta, in: KOLOR - Journal on Moving Communities 6: 2, 4-31. 
Neal, Andrew 2009: Securitization and Risk at the EU Border: The Origins of FRONTEX, in: Journal of Common Market Studies 47: 2, 333-356.

OBC - Osservatorio Balcani e Caucaso 2005: Tragedia Kater, una sentenza deludente, in: http://www.osservatoriobalcani.org/article/articleview/4056/1/41/; 22.6.2010.

Shore, Cris 2006: 〉Government without Statehood ? Anthropological Perspectives on Governance and Sovereignty in the European Union, in: European Law Journal 12: 6, 709-724.

Texeire, Fiona 2006: At the Gate of Fortress Europe: Irregular Immigration and Malta, Rennes.

Weinzierl, Ruth/Lisson, Urszula 2007: Grenzschutz und Menschenrechte. Eine europarechtliche und seerechtliche Studie (herausgegeben von: Deutsches Institut für Menschenrechte), Berlin, in: http://www.institut-fuer-menschenrechte.de/up loads/tx_commerce/studie_grenzschutz_und_menschenrechte.pdf; 30.04.2013.

\section{Die Autorin}

Dr. Silja Klepp ist wissenschaftliche Mitarbeiterin am artec | Forschungszentrum Nachhaltigkeit der Universität Bremen und Trägerin des Christiane-Rajewsky-Preises 2012.

E-Mail: klepp@uni-bremen.de 